\title{
Feasibility of Fully Automated Motion Compensated Overlay for Transcatheter Aortic Valve Implantation
}

\author{
Nick Assink, MSc ${ }^{1}$, Maria-Louisa Izamis, $\mathrm{PhD}^{2}$, Olivier Nempont, PhD ${ }^{3}$, Marco Verstege, PDEng ${ }^{1}$, \\ Cherif P. Sahyoun, PhD', Alexander Haak, PhD', John D. Carroll, MD4, John C. Messenger, MD4, \\ Gerhard Schymik, MD5, Navid Madershahian, MD', Thorsten C. Wahlers, MD ${ }^{6}$, Peter Eshuis, PhD, ${ }^{1 *}$ \\ ${ }^{1}$ Philips Healthcare, Best, The Netherlands \\ ${ }^{2}$ Philips Research, Eindhoven, The Netherlands \\ ${ }^{3}$ Philips Research, Paris, France \\ ${ }^{4}$ Division of Cardiology, University of Colorado Hospital, Aurora, Colorado, USA \\ ${ }^{5}$ Municipal Hospital Karlsruhe, Karlsruhe, Germany \\ ${ }^{6}$ Department of Cardiac and Thoracic Surgery, Cologne University Heart Center, Cologne, Germany
}

\begin{abstract}
Background: Automated motion compensation of aortic root overlay on fluoroscopy during transcatheter aortic valve implantation (TAVI) could ensure accurate device positioning at minimal contrast cost, thereby reducing complication rates.

Objectives: To describe the feasibility of software that automatically compensates for cardiac and respiratory motion on X-ray, which may allow greater device control during TAVI.

Methods: Twenty four TAVI cases (25,607 frames) from four independent institutions using either the Medtronic CoreValve $(n=8)$ or Edwards Sapien valve $(n=16)$ were post-processed with the software. For each case, the algorithm applied three steps: (i) Generation of an anatomical roadmap using X-ray (Vascular Outlining, or VO) or 3D segmentation of CT data, (ii) Correlation to pigtail catheter, and (iii) Real-time motion compensation.

Results: VO motion compensation was activated $84 \%$ of all frames yielding a relative displacement error of $-1.09 \pm 2.65 \mathrm{~mm}$. Similarly, CT-aided motion compensation was activated $84 \%$ of frames yielding a relative displacement error of $-0.77 \pm 2.92 \mathrm{~mm}$.
\end{abstract}

(C) 2018 Journal of Structural Heart Disease Published by Science International Corp. ISN 2326-4004

Fax +1 2037853346

E-Mail: jshd@scienceinternational.org

http://structuralheartdisease.org/
Conclusions: We have shown feasibility of the first fully automated motion compensation method for real-time continuous visualization of the target aortic anatomy during TAVI procedures. Our method has the potential to improve valve positioning accuracy and reduction in deployment variability.

Copyright $\odot 2018$ Science International Corp.

Key Words

Aortic stenosis • TAVI • TAVR • Imaging modalities • Noninvasive imaging

\section{Introduction}

With over 250,000 procedures conducted worldwide in the last decade, transcatheter aortic valve implantation (TAVI) has gained widespread acceptance for the treatment of aortic valve disease [1]. As outcomes continue to improve, TAVI is expected to be performed in younger, lower-risk patients [2] and will grow the number of procedures further. Correct positioning of the artificial valve is crucial forTAVI outcome [3]. Current implantation of prosthetic aortic valves

* Corresponding Author:

Peter Eshuis, PhD

Philips Healthcare

Veenpluis 6, 5684 PC Best, The Netherlands

Tel. +31 (0)40 2763681; E-Mail: peter.g.eshuis@philips.com 
i. Anatomical roadmap generation

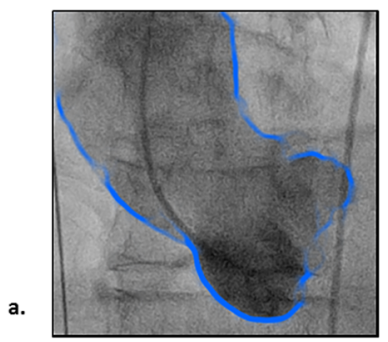

b.

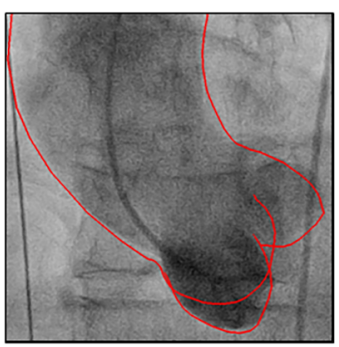

ii. Correlate anatomical roadmap to pigtail catheter

c.

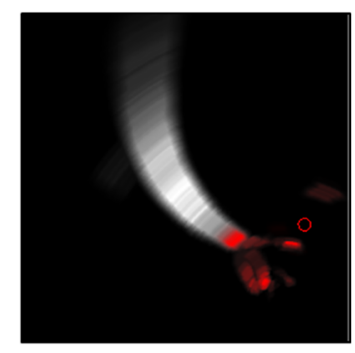

d.

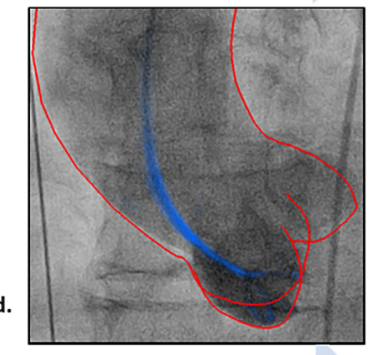

iii. Live motion compensation
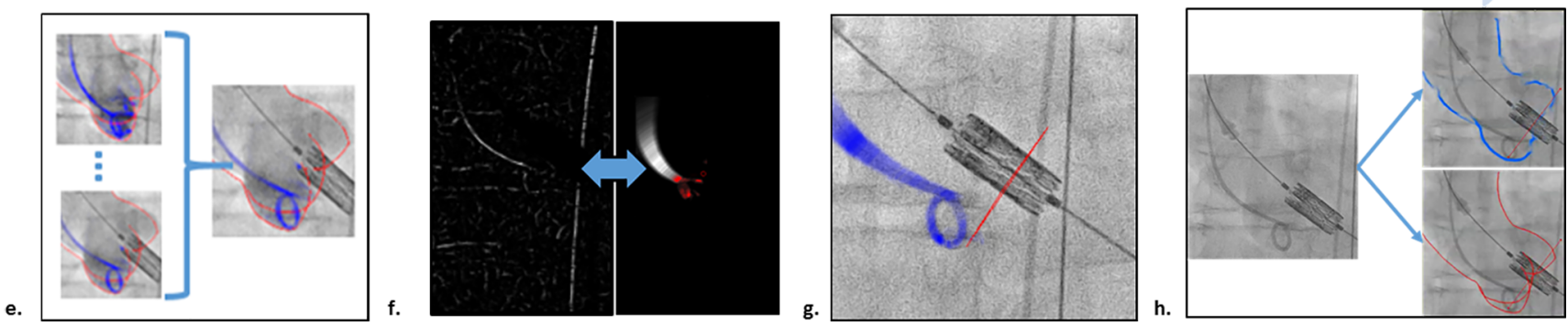

Figure 1. The three steps of motion compensation: i. Anatomical roadmap generation: (Panel A) Vascular Outlining (VO) based on the angiographic image, (Panel B) Computed-Tomography (CT) segmentation registered to the angiographic image. ii. Correlate anatomical roadmap to pigtail catheter: (Panel C) The reference map for the pigtail is extracted, (Panel D) The spatial relation between the pigtail reference map (blue) and the anatomical roadmap (red) is set. iii. Live motion compensation: (Panel E) The pigtail reference map best matching the current pigtail shape is selected, (Panel F) Live fluoroscopic image is filtered (left) and matched to the pigtail reference map (right), (Panel G) Fluoroscopic view of the matching result, (Panel H) The transformation is applied to the anatomical roadmap resulting in a dynamic motion-compensated roadmap, either VO (blue) or CT (red).

outside the optimal depth range still occurs in $21 \%$ of the cases [4], resulting in high-degree atrioventricular block (10-30\%) and paravalvular leak (4-35\%) [5]. We have created a fully-automated software that enables anatomical roadmap overlays on live fluoroscopic images compensated for cardiac and respiratory motion without workflow disruptions, which may allow for greater control over valve placement. This paper describes how our technology works and reports on the results of the feasibility study performed.

\section{Method}

Our algorithm comprises three steps:

i. Anatomical roadmap generation. Angiograms with contrast injections are automatically identified and the frame best opacifying the aortic root is selected by the algorithm, upon which two types of anatomical roadmaps are generated:

1. Vascular Outlining (VO): The outline of contrast is detected in the X-ray image (Figure 1a).

2. Computed Tomography (CT) aided: The automatic CT segmentation $[6,7]$ is registered to the angiographic image (Figure $1 \mathrm{~b}$ ).

ii. Correlate anatomical roadmap to the pigtail catheter. The pigtail catheter is routinely locked in an aortic valve cusp and its motion reflects overall aortic valve motion. The software searches for the pigtail catheter (Figure 1c) and sets the spatial relationship with respect to the anatomical roadmap (Figure 1d). This correlation process is performed for all angiograms producing a series of references (Figure 1e).

iii. Live motion compensation. Each live fluoroscopic image is filtered to enhance pigtail-like objects, which is then matched to the references (Figure 
Table 1. $\mathrm{VO}$ and $\mathrm{CT}$-aided motion compensation results.

\begin{tabular}{|c|c|c|c|c|c|c|c|c|}
\hline & \multirow{2}{*}{\multicolumn{3}{|c|}{ VO MC }} & \multirow{2}{*}{\multicolumn{3}{|c|}{ CT-aided MC }} \\
\hline & & & & & & & & \\
\hline Valve type & $\begin{array}{l}\text { Pigtail catheter } \\
\text { cusp position }\end{array}$ & $\begin{array}{l}\text { Number of } \\
\text { cases }\end{array}$ & $\begin{array}{c}\text { Frames with } \\
\text { activated MC } \\
\text { (\%) }\end{array}$ & $\begin{array}{c}\text { Relative } \\
\text { displacement } \\
\text { error }(\mathrm{mm})\end{array}$ & $\begin{array}{l}\text { Absolute } \\
\text { displacement } \\
\text { error }(\mathrm{mm})\end{array}$ & $\begin{array}{c}\text { Frames with } \\
\text { activated } \\
\text { MC (\%) }\end{array}$ & $\begin{array}{c}\text { Relative } \\
\text { displacement } \\
\text { error }(\mathrm{mm})\end{array}$ & $\begin{array}{l}\text { Absolute } \\
\text { displacement } \\
\text { error }(\mathrm{mm})\end{array}$ \\
\hline CoreValve & Lowest & 8 & 82 & $-1.10 \pm 2.61$ & 2.00 & 85 & $-1.13 \pm 2.91$ & 2.15 \\
\hline \multirow{2}{*}{ Sapien } & Lowest & 4 & 87 & $0.09 \pm 2.56$ & 1.97 & 98 & $-0.15 \pm 2.45$ & 1.50 \\
\hline & Middle & 12 & 89 & $-1.24 \pm 2.72$ & 2.71 & 80 & $-0.09 \pm 3.02$ & 2.48 \\
\hline Total & - & 24 & 84 & $-1.09 \pm 2.65$ & 2.24 & 84 & $-0.77 \pm 2.92$ & 2.22 \\
\hline
\end{tabular}

$1 \mathrm{f}-\mathrm{g})$. The anatomical roadmap is then transformed accordingly to obtain a real-time dynamic motion-compensated roadmap (Figure 1h). Motion compensation is deactivated automatically if the pigtail catheter is obstructed, such as by the TEE probe, and activated when the pigtail catheter is successfully found again.

The live motion compensation is real-time up to 30 frames per second using an Intel ${ }^{\circledR}$ Xeon E5-1620 v3 CPU 3.50GHz.

\section{Automatic Motion Compensation Evaluation Protocol}

The use of a motion compensated overlay occurs during the device positioning and deployment phase, so we post-processed X-ray data of 24 cases during this phase to evaluate the algorithmic performance. None of these datasets were used for algorithm development.

First, the percentage of frames in which motion compensation was correctly activated by the algorithm was determined. Secondly, the relative and absolute displacement error were determined for every X-ray frame by comparing the manually annotated pigtail catheter and aortic root position with the algorithmic roadmap position, where a negative relative displacement error denotes deeper positioning by the algorithm. Continuous variables were given as mean \pm standard deviation and categorical variables were given as percentages.

\section{Results}

For all 24 cases (25,607 frames) we evaluated the performance of motion compensation (Table 1). VO motion compensation was activated $84 \%$ of all frames yielding a relative displacement error of -1.09 $\pm 2.65 \mathrm{~mm}$ and $2.24 \mathrm{~mm}$ absolute displacement error. CT-aided motion compensation was activated $84 \%$ of all frames yielding a relative displacement $-0.77 \pm$ $2.92 \mathrm{~mm}$ and $2.22 \mathrm{~mm}$ absolute displacement error.

The relative and absolute displacement error increased for the larger and hence more obstructive CoreValve and also increased when the pigtail catheter was positioned in the more obstructive middle position (Table 1). Overall VO and CT-aided motion compensation demonstrated similar performance.

\section{Discussion}

We have used the pigtail catheter as a contrast-independent landmark for motion compensation during TAVI without any need for software interaction. To our knowledge, only one approach has successfully tracked the aortic valve plane by using the calcifications on the aortic valve as contrast-independent landmarks [8]. A clinical trial correlated this approach with a promising reduction in the incidence of conduction disorders [9]. The feasibility of the approach was limited by the need to manually annotate the calcifications after every repositioning of the C-arm. Additionally, not every patient may have sufficient visible calcifications [10]. All currently available CT fusion solutions provide static overlays only.

Of the two motion compensation methods evaluated: VO has the advantage of requiring only a well-contrasted aortic root angiogram representing the current aortic anatomical situation. CT-aided motion compensation provides a richer $3 \mathrm{D}$ view, with the ability to integrate pre-procedural planning in the live roadmap. 
A major limitation of the study design was the post-processing of data. Actual clinical use of motion compensation is needed to determine the impact of the motion compensated anatomical roadmap on valve positioning. Another limitation of the technology is its dependence on the pigtail catheter maintaining its position locked in one of the aortic valve cusps. It is important not to lose this position as the device is advanced, as the relationship between the pigtail and the valve plane is assumed constant. Whereas this is common in clinical practice during valve positioning, the pigtail is typically pulled in the last phases of deployment, implying that the motion compensated overlay may not be used for guidance if any final adjustments are required. Further studies are warranted to examine whether these limitations are clinically acceptable. Of note, the live overlay is automatically disabled when detecting pigtail retrieval, to avoid erroneous guidance.

The implications of this work are perhaps greatest for enhancing the learning curve amongst new operators and for physicians performing TAVIs on lower-risk patients with potentially fewer X-ray-visible anatomic landmarks. Prospective studies of impact of this technology on contrast usage and positioning accuracy are warranted.

\section{Conclusion}

We have shown feasibility of the first fully automated motion compensation method for real-time continuous visualization of the target aortic anatomy during TAVI procedures. Our method has the potential to improve valve positioning accuracy and reduction in deployment variability and contrast usage.

\section{Conflict of Interest}

- Nick Assink - Master student located at Philips Healthcare)

- Maria-Louisa Izamis - Employee of Philips Healthcare

- Olivier Nempont - Employee of Philips Healthcare

- Marco Verstege - Employee of Philips Healthcare

- Cherif P. Sahyoun - Employee of Philips Healthcare

- Alexander Haak - Employee of Philips Healthcare

- John D. Carroll - Research funding and in-kind support from Philips Healthcare

- John C. Messenger - Research funding and in-kind support from Philips Healthcare

- Gerhard Schymik - In-kind support from Philips Healthcare

- Navid Madershahian - In-kind support from Philips Healthcare

- Thorsten C. Wahlers - In-kind support from Philips Healthcare

- Peter G. Eshuis - Employee of Philips Healthcare

Comment on this Article or Ask a Question

\section{References}

1. Cribier A. Historical perspective: 10th year anniversary of TAVI. Eurolntervention. 2012;8:Q15-17. DOI: 10.4244/EIJV8SQA4

2. Babaliaros V, Devireddy C, Lerakis $S$, Leonardi $\mathrm{R}$, Iturra SA, Mavromatis $\mathrm{K}$ et al. Comparison of transfemoral transcatheter aortic valve replacement performed in the catheterization laboratory (minimalist approach) versus hybrid operating room (standard approach): outcomes and cost analysis. JACC: Cardiovascular Interventions. 2014;7:898-904. DOI: 10.1016/j. jcin.2014.04.005

3. Cao C, Ang SC, Vallely MP, Ng M, Adams $\mathrm{M}$, Wilson M. Migration of the transcatheter valve into the left ventricle. Ann Cardiothoracic Surg. 2012;1:243-244. PMID: 23977502
4. Muñoz-García AJ, del Valle R, Trillo-Nouche R, Elízaga J, Gimeno F, Hernández-Antolín $\mathrm{R}$, et al. The Ibero-American transcatheter aortic valve implantation registry with the CoreValve prosthesis. Early and long-term results. Int J Cardiol. 2013;169:359-365. DOI: 10.1016/j.ijcard.2013.09.006

5. Yan TD, Cao C, Martens-Nielsen J, Padang R, Ng M, Vallely MP, Bannon PG (2010). Transcatheter aortic valve implantation for high-risk patients with severe aortic stenosis: a systematic review. The Journal of thoracic and cardiovascular surgery. 2010;139:1519-1528. DOI: 10.1016/j. jtcvs.2009.08.037

6. Ecabert $\mathrm{O}$, Peters J, Schramm H, Lorenz C, von Berg J, Walker MJ, et al. Automatic model-based segmentation of the heart in CT images. IEEE Trans Med Imaging, 2008;27:1189-1201. DOI: 10.1109/ TMI.2008.918330

7. Wächter I, Kneser R, Korosoglou G, Peters J, Bakker NH, vd Boomen R, Weese J. Patient specific models for planning and guidance of minimally invasive aortic valve implantation. Int Conf on MICCAI. 2010;September:526-533. PMID: 20879271

8. Dvir D, Kornowski R. Percutaneous aortic valve implantation using novel imaging guidance. Cathet Cardiovasc Int. 2010;76:450-454. DOI: 10.1002/ccd.22362

9. Codner P, Lavi I, Malki G, Vaknin-Assa H, Assali A, Kornowski R. C-THV measures of self-expandable valve positioning and correlation with implant outcomes. Catheterization and Cardiovascular Interventions. 
2014;84:877-884. DOI: 10.1002/ccd.25594

10. Xia Y, Hussein S, Singh V, John M, Wu Y, Chen T. Context region discovery for automatic motion compensation in fluoroscopy. International journal of computer assisted radiology and surgery. 2016;11:977-985. DOI: 10.1007/s11548-016-1362-y
Cite this article as: Assink N, Izamis $M$, Nempont $O$, Verstege $M$, Sahyoun $C_{2}$ Haak A, Carroll JD, Messenger JC, Schymik G, Madershahian N, Wahlers TC, Eshuis P. Feasibility of Fully Automated Motion Compensated Overlay for Transcatheter Aortic Valve Implantation. Structural Heart Disease. 2018;4(5):207211. DOI: https://doi.org/10.12945/j. jshd.2018.050.17 